\title{
AGENT-BASED MODELING FOR HOUSEHOLD LEVEL HURRICANE EVACUATION
}

\author{
Bo Zhang \\ Wai Kin (Victor) Chan \\ Department of Decision Sciences \& Engineering Sys- \\ tems \\ Rensselaer Polytechnic Institute \\ Troy, NY 12180, U.S.A.
}

Satish V. Ukkusuri

\author{
Department of Civil \& Environmental Engineering \\ Rensselaer Polytechnic Institute \\ Troy, NY 12180, U.S.A.
}

\begin{abstract}
In this paper, we present an agent-based model for hurricane evacuation which captures the interactions among evacuees during the evacuation process. Both normal and greedy household agents are considered. The normal agents simply follow the shortest route toward their destinations regardless of the congestion on each route segment. The greedy agents on the other hand make choices between the shortest route and the least congested route according to a certain probabilistic rule, taking into account the evolving network conditions. In the experiments, we simulate different scenarios on a test network and present the results and analysis. The simulation results indicate that greedy agents can sometimes reduce the individual evacuation times by detouring and avoiding congestions, but the greedy behavior makes the whole evacuation inefficient.
\end{abstract}

\section{INTRODUCTION}

Based on history records and observed trends, hurricanes have been becoming more intense and frequent. The population of the coastal states is steadily growing while the transportation infrastructures of these areas fail to keep pace with this growth (Dow and Cutter 2002). Therefore, hurricane evacuations are increasingly becoming problematic and complex. This is obvious in recent years. For instance, in 2005 New Orleans was devastated by Hurricane Katrina and the Houston area was threatened by Hurricane Rita. Typically, evacuations are characterized by traffic jams and chaos in which people spend 10 to 20 hours waiting on the roads. In order to reduce or prevent unnecessary traffic jams during evacuations, it is crucial that coastal states predefine an effective evacuation plan to minimize the human suffering. While some of these plans already exist, most of them do not incorporate the decision making of drivers accurately. Traffic jams and chaos during evacuations are mainly caused by human behaviors in competing for resources or space to survive. Understanding these competing behaviors at household level in the threatened areas, such as traffic interactions, is one of the main concerns for government agencies in planning for emergency evacuations. Nevertheless, due to the complex nature of evacuations, designing a viable and efficient plan would be difficult without the help of analytic and/or experimental tools.

Agent-based simulation is a computational methodology to model systems comprised of interacting autonomous agents situated in an artificial environment (Macal and North 2005). These autonomous agents are self-directed objects with the capabilities of making decision and reacting to the environment. They also have the ability to learn and adopt based on their goals and interactions with each other. Such a framework is particularly suitable for simulating individual behaviors and exploring emergent collective phenomena in evacuation. To capture some of the phenomena and complexities during evacuations, we develop an agent-based model for hurricane evacuations to simulate household-level evacuations and the resulting interactions among evacuees.

In particular, in this paper, we present a preliminary agent-based model for hurricane evacuations. This model is a multiagent system of a representative transportation network. It tries to capture the human traffic behavior and their interactions during an evacuation process. The rest of the paper is organized as follows. Section 2 reviews related work. Section 3 describes the architecture of the model and the definition of agent behavior. Section 4 provides the experimental results of different evacuation scenarios. Section 5 outlines the conclusions and future work.

\section{RELATED WORK}

There is a considerable amount of research in models that support hurricane evacuation. Cova and Johnson (2003) presents a network flow model for lane-based evacuation routing plans in a transportation network. This model is an integer extension 
of the minimum cost flow problem and the mixed-integer programming is used to obtain optimal routing plans for the whole traffic network. But this model has multiple objectives, total evacuee travel distance and the number of intersection conflicts. People have to trade travel distance against conflicts manually. Although LP methods can generate optimal evacuation plans, they are very expensive to implement on large urban traffic networks. However, as to hurricane evacuations, the major parts of evacuation routing are in rural areas. In practice, LP methods are usually used in scenarios that have small sized networks such as, in building evacuations (Kim et al. 2007). It is difficult for LP models to get the results when networks are in large size. Lu, et al. (2005) use general shortest path algorithms and extends them by incorporating capacity constraints. It models capacity as a time series to account for the time dependent nature of the networks. This method can generate evacuation route effectively used in medium-sized networks, it might not scale up efficiently to an evacuation scenario that involves millions of evacuees and a transportation network of hundreds of thousands of nodes. Moreover, this model has several assumptions that may not be the truth. For example, the travel time on edges must be constant, it cannot solve if travel time is variable. Also, edge travel time must preserve FIFO (First-In First-Out) property which may not be the real case. This model cannot capture the traffic interactions. This problem will limit the practicability of traffic models in real evacuation planning.

Currently, applications of agent-based technologies has been presented abundantly for many scenarios and problems, such as reproducing human behavior, pedestrian and vehicular simulation. TRANSIMS and MATSim are two representative agent-based tools in traffic simulation. TRANSIMS (TRANSIMS 2009) is able to simulate the second-by-second movements of every person and every vehicle and analyze on a micro level to provide information for every simulated traveler. MATSim (MATSim 2009) provides a toolbox to implement large-scale agent-based transport simulations. Although TRANSIMS and MATSim can give relatively accurate and complete information on traffic impacts, both of the tools cannot simulate the human and social behaviors during emergency evacuations. Researchers have built plenty of evacuation models to understand the human and social behaviors and test their influences during emergency evacuations. Pelechano et al. (2005) describe an architecture of a crowd simulation system which incorporates a psychological model to obtain emergent behaviors of humans. This framework combines a psychological model with a crowd simulation system. The crowd simulation system manages the local motion of individuals in a room, the way-finding process, and implements the agent bodies, actions and results using communication and roles. The psychological model handles the human behavioral models for physiology, stress, perception and emotion. This crowd simulation model is useful in understanding human behaviors during building evacuations. Kagaya et al. (2005) develop a multi-agent model based on behavior-oriented agent systems. This study first carried out a questionnaire survey for the inhabitants to construct the production rules of the attributive groups, then developed a multiagent system model for evacuation based on the set of production rules. This multi-agent model is tested by simulating a district earthquake evacuation in Japan. It is shown to reproduce the human behaviors and their interactions during an earthquake strike. This model, however, pays less attention to the resulting traffic interactions during an evacuation, such as route choice and congestion.

In view of this background, this study mainly focuses on dynamic traffic condition among the agents during the evacuation process. The objectives of this study is to build the evacuation simulation model based on the action rules of household agents and capture the traffic behaviors and interactions between them. Using this evacuation simulation model, we test and analyze different scenarios, and grasp the characteristics and effects of human traffic behaviors during the evacuation.

\section{MODEL DESIGN}

The simulation model is created using the agent-based toolkit, Repast Simphony (North et al. 2007). Our model includes four components: network constructor, agent constructor, displayer, and simulation context. We briefly discuss these four components in the following:

(1) Network Constructor: the purpose of this module is to generate transportation networks from shape files. The shape files here include three types, Point, Poly Line (which generates roads), and Polygon (which generates houses). The network includes roads on which evacuee agents travel, intersections, and houses which are the origins and destinations of evacuee agents.

(2) Agent Constructor: this module generates agents on the basis of action rules we define. We can create household agents either manually or based on the locations provided by the shape files of Point type.

(3) Displayer: this module primary displays the animation of simulation dynamics and results.

(4) Simulation Context: this module is the global environment. In the environment, each autonomous agent proceeds to its destination subject to certain imposed constraints and interacts with the environment and other agents. It also provides real-time information about other household agents as well as the transportation network during the simulation.

Our simulation model is a decentralized multi-agent system. That is, there is no central system control mechanism. 


\subsection{Household Agents}

Our overall objective is to effectively integrate household decision making into the evacuation modeling process so that different policy scenarios can be analyzed and evaluated. In this paper, we will focus on developing a preliminary agent-based model with a simple yet representative network and behavioral rules for household decision making in evacuation. In our model, each household is an agent. In a real system, after a warning is issued, a household will spend a certain amount of time before deciding to evacuate or not. We assume that a collective decision making process is used by each household to arrive at the final decision: to evacuate or not. For simplicity, in the current study we only model those who eventually evacuate. Also, we assume that each household uses only one vehicle to execute their evacuation. When the evacuation starts, each household agent starts from its home and enters the traffic network. Because houses may not position right at a main road, the agents' first task is to proceed to the nearest main road through a private path (such as a drive way) at a constant low speed. Upon reaching a main road, the agent can gradually speed up to the speed limit if the traffic on the road allows.

There are two types of agents in our model: normal agents and greedy agents. We explain how each type of agents evacuate. Both types of agents know their destinations before evacuation. The action rules of normal agent are shown in Figure 1. A normal agent chooses the route that has the smallest travel distance to the destination. While traveling, the agent needs to constantly check whether there are other vehicles in the near front. If no, it can speed up; otherwise it has to slow down to the same speed as its nearest front vehicle to avoid collisions. Slowing down is also required when an agent is approaching an intersection.

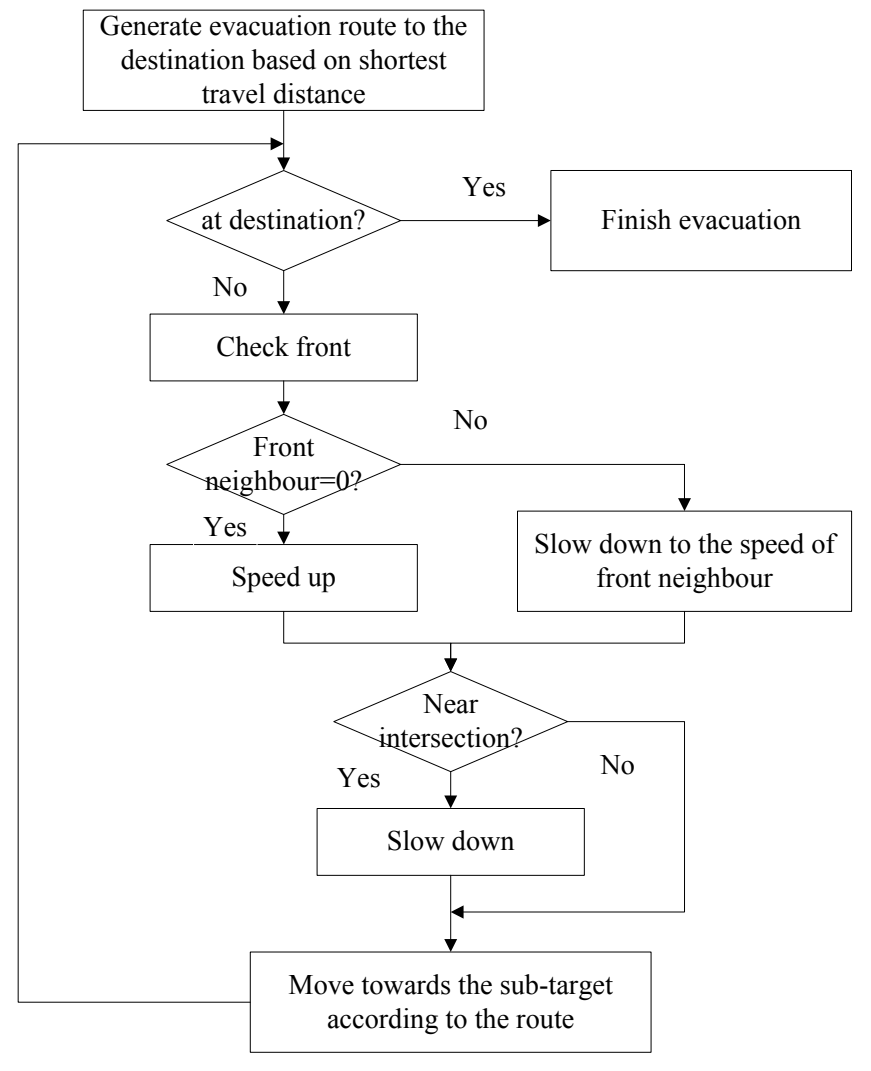

Figure 1: Action rules of normal agents

Figure 2 describes the action rules of a greedy agent. When a greedy agent is approaching an intersection, it perceives the congestion level on all connected road segments. If the congestion level on its original shortest route exceeds a pre-defined tolerance limit, the greedy agent will choose a new connected road segment (which is the least congested) and re-calculate a new evacuation route starting from this new selected road segment. In summary, a normal agent does not change its route after it starts evacuation, while a greedy agent may adaptively change its route to avoid congestion. Because the shortest route determined at the beginning of the evacuation has the shortest travel distance, any new routes selected by a greedy agent during evacuation are likely to detour to the destination with a longer travel distance. To avoid constantly changing the routes, a tolerance limit is imposed in greedy agents' decision of selecting new routes. In particular, if the new route has a much longer 
distance than the old route or the number of route changes has reached a certain predefined limit, a greedy agent will stay in its original route.

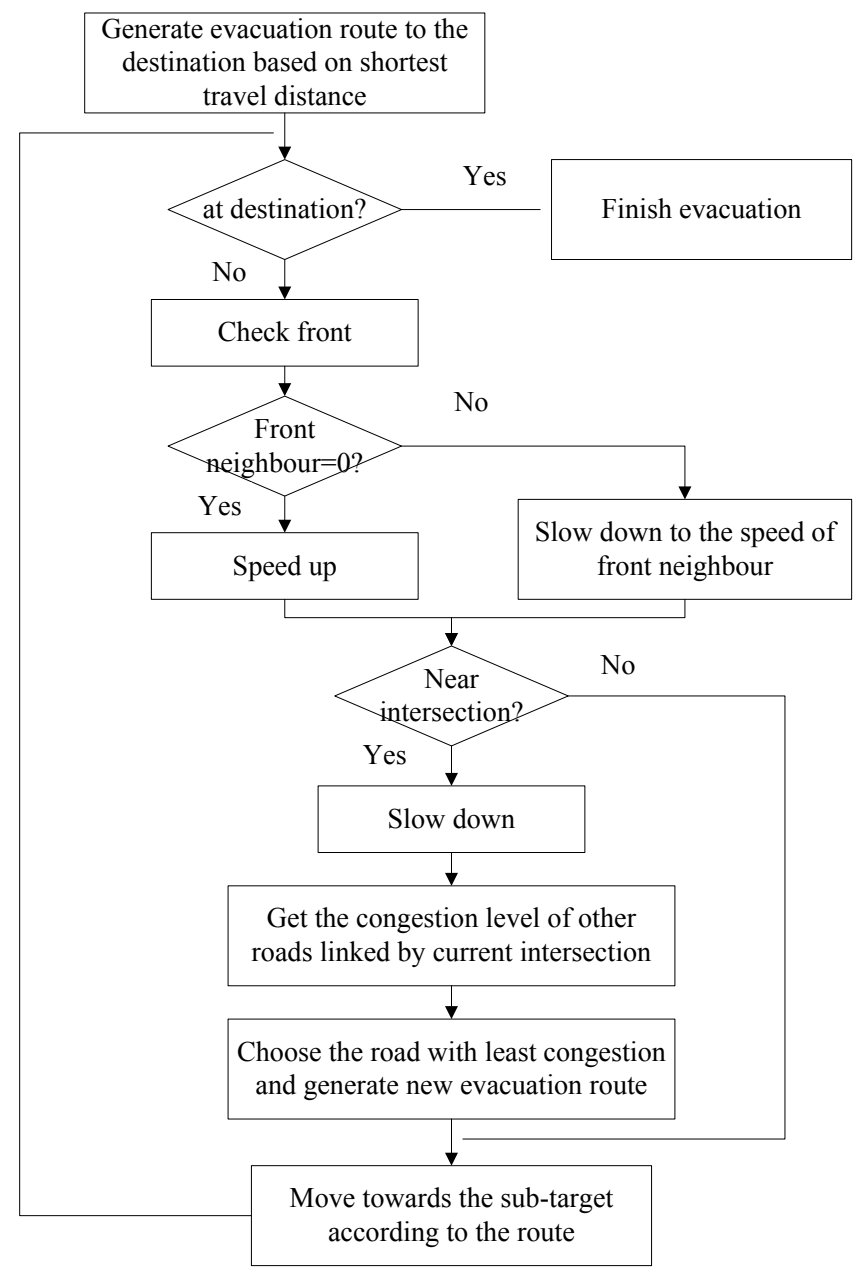

Figure 2: Action rules of greedy agents

\section{EXPERIMENTAL RESULTS AND ANALYSIS}

In this section, we present experimental studies on different scenarios of a test network. The goal is to examine how the proportion of normal and greedy agents affects the evacuation efficiency. Here, evacuation efficiency is measured by total evacuation time which starts from the time at which the first agent leaves its house until the time at which the last agent arrives at the destination. In each scenario, we record the individual evacuation times of all agents and compute their minimum, maximum, average, and standard deviation. In our simulation experiments, the total number of agents ranges from 50 to 1000 . The greedy agent is further separated into two cases: $50 \%$ greedy and $100 \%$ greedy. The $50 \%$ greedy means that when a greedy agent is about to make a decision (i.e., near an intersection and the congestion level on its original route is larger than the threshold), it has $50 \%$ possibility to choose the least congested road and generate new evacuation route. The agent will stay in the original route with the remaining $50 \%$ of chance. The $100 \%$ greedy case means that the greedy agent will for sure behave greedily. Both cases are restricted by the same tolerance limit. We define the tolerance limit of greedy agents as follows: if the new route is two times as the length of the old route or the number of route changes is larger than 10, a greedy agent will stay in its original route.

Figure 3 shows the test network and one evacuation route of an example agent. Agents start from their house, enter the main roads, and travel to the destination to evacuate from the network. In our model, we use a graph to represent the transportation network. The Dijkstra's shortest path algorithm is used to obtain the shortest path between any pair of nodes in the 
graph. However, the origin and destination of an agent may not be exactly on a node; they can be any point on an edge. To handle this issue, we consider the two nodes closest to the origin and the two nodes closest to the destination. We then use the Dijkstra's shortest path algorithm to obtain four shortest routes between the two nodes closest to the origin and the two nodes closest to the destination. The shortest one among these four routes will be the actual shortest path between the origin and destination.

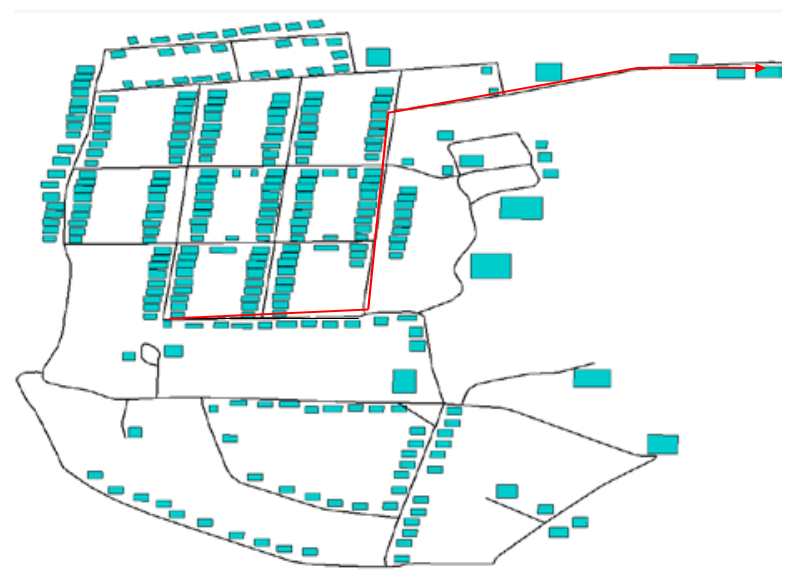

Figure 3: Test network

Figure 4 illustrates how the evacuation time changes in the number of normal agents. It can be seen that when the number of normal agents increases, the minimum, average, and maximum evacuation times only alter slightly. However, the standard deviation of all the individual evacuation times increases with the number of agents in the simulation environment.

Figure 5 illustrates the experimental results with 1000 agents with the proportion of greedy agents ranging from $0 \%$ to $100 \%$ at an increment of $10 \%$. This amounts to a total of 11 experiments. All the greedy agents are $100 \%$ greedy. In each experiment, the simulation is executed by randomly choosing the corresponding percentage of agents as greedy ones and different results are obtained by every execution, so that the average values of twenty simulation executions are adopted as the evacuation results. From Figure 5, we can observe that the evacuation time tends to increase in overall trend as the percentage of greedy agents increases Although sometimes the changing route and detouring of greedy agents can reduce their individual evacuation times, the greedy behavior will increase the total evacuation time.

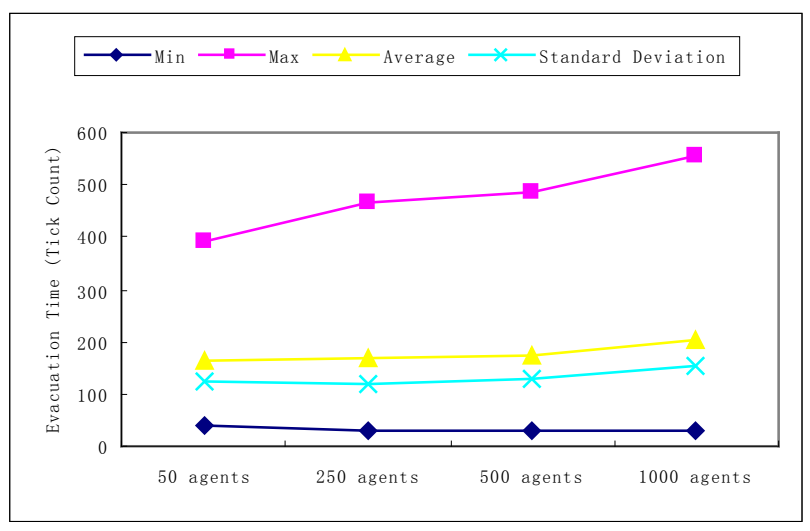

Figure 4: Evacuation time vs. number of agents

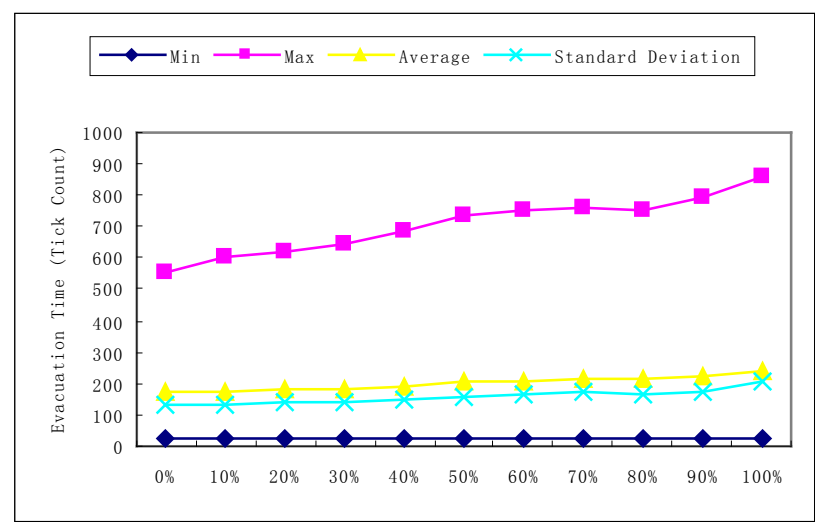

Figure 5: Evacuation time vs. percentage of greedy agents

Figure 6 and 7 illustrate the minimum, maximum, average, and standard deviation of the two cases: $50 \%$ greedy and $100 \%$ greedy. In both cases, the total number of agents is 1000 . From Figure 6 , we can observe that the minimum, maximum, and average values of the $50 \%$ greedy case are no greater than those of the $100 \%$ greedy case in most conditions. These results suggest that being less greedy can reduce the total evacuation time, especially when there are a large number of greedy 
agents. Figure 7 shows that when greedy agents are in a high proportion, the standard deviation of the $50 \%$ greedy case is larger than that of the $100 \%$ greedy case.

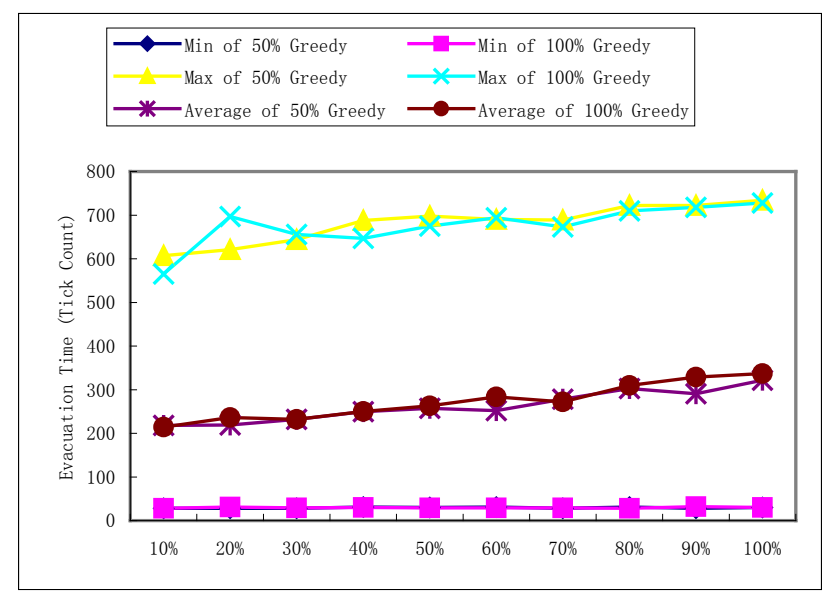

Figure 6: The changes of Min, Max, and Average evacuation time of $50 \%$ greedy vs. $100 \%$ greedy

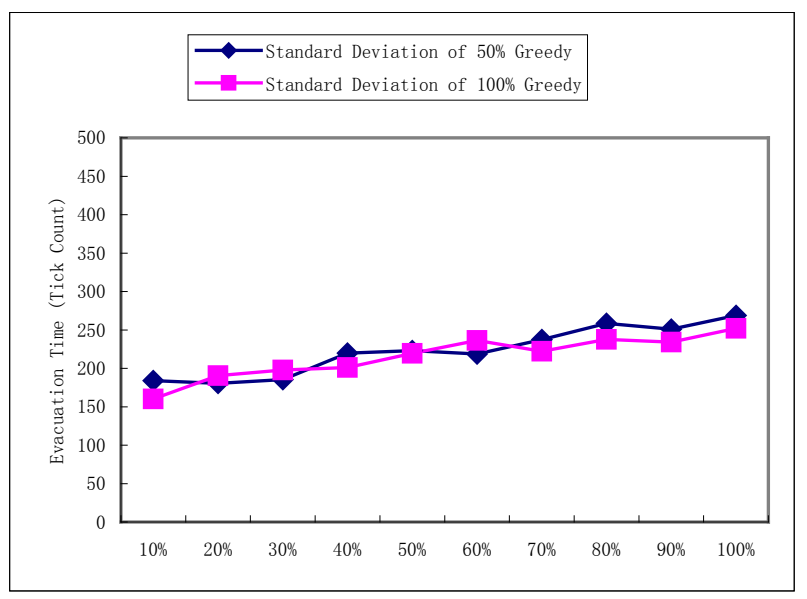

Figure 7: Standard deviation of evacuation time of $50 \%$ greedy vs. $100 \%$ greedy

\section{CONCLUSIONS AND FUTURE WORK}

In this paper, we present an agent-based evacuation model and invest the dynamic traffic behaviors and agents' interactions during evacuations. We classify two types of agents, normal agents and greedy agents and build the action rules based on their evacuation behaviors. We execute several simulations on different scenarios and test the influence of the behavior of greedy agents. The experimental results suggest that the greedy behavior can make the whole evacuation inefficient especially when the percentage of greedy agents is high, even though being greedy can sometimes reduce the individual evacuation times by detouring and avoiding congestions. Future work include examining the passing-over behavior and accident conditions during the evacuation. We can also refine the model and consider more realistic evacuation actions in the future.

\section{ACKNOWLEDGMENTS}

This work is supported by NSF project Collaborative Proposal: DRU: Incorporating Household Decision Making and Dynamic Transportation Modeling in Hurricane Evacuation: An Integrated Social Science-Engineering Approach, with project No. 0826874.

\section{REFERENCES}

Cova, T.J., and Johnson, J. P. 2003. A network flow model for lane-based evacuation routing. Transportation Research Part A, 37: 579-604.

Dow, K., and Cutter, S. L. 2002. Emerging hurricane evacuation issues: Hurricane Floyd and South Carolina. Nat. Hazards Rev., 3(6): 12-18.

Kagaya, S., Uchida, K., Hagiwara, T., and Negishi, A. 2005. An application of multi-agent simulation to traffic behavior for evacuation in earthquake disaster. Journal of the Eastern Asia Society for Transportation Studies, 6: 4224-4236.

Kim, S., George, B., and Shekhar, S. 2007. Evacuation route planning: scalable heuristics. In Proceedings of the 2007 International Symposium on Advances in Geographic Information Systems.

Lu, Q., George, B., and Shekhar, S. 2005. Capacity constrained routing algorithms for evacuation planning: a summary of results. In Proceedings of the 2005 International Symposium on Advances in Spatial and Temporal Databases.

Macal, C. M., and North, M. J. 2005. Tutorial on agent-based modeling and simulation. In Proceedings of the 2005 Winter Simulation Conference, eds. M. E. Kuhl, N. M. Steiger, F. B. Armstrong, and J. A. Joines, 2-15. Piscataway, New Jersey: Institute of Electrical and Electronics Engineers, Inc.

MATSim. 2009. MATSim home page, <http://www.matsim.org/> [accessed February 19, 2009]. 
North, M. J., Howe, T. R., Collier, N. T., and Vos, J. R. 2005. The Repast Simphony runtime system. In Proceedings of the Agent 2005 Conference on Generative Social Processes, Models, and Mechanisms.

Pelechano, N., O’Brien, K., Silverman B., Badler, N. 2005. Crowd simulation incorporating agent psychological models, roles and communication. In Proceeding of the 2005 International Workshop on Crowd Simulation.

TRANSIMS. 2009. TRANSIMS home page, <http://www.transims-opensource.org/> [accessed March 7, 2009].

\section{AUTHOR BIOGRAPHIES}

BO ZHANG is a Ph.D. student of the Department of Decision Sciences and Engineering Systems at Rensselaer Polytechnic Institute. He received a bachelor's degree and a master's degree in computer science from Northeastern University. His current research are agent-based modeling and simulation on hurricane evacuation. His e-mail address is <zhangb5@rpi.edu>.

SATISH V. UKKUSURI is an Assistant Professor of Transportation in the Civil and Environmental Engineering at Rensselaer Polytechnic Institute. He received his Ph.D. from the University of Texas at Austin in 2005. His research interests are in dynamic transportation modeling, logistics, disaster management and vehicular ad hoc networks. He serves as the Overview Paper Editor of Transportation Research Part-C and an Associate Editor of Networks and Spatial Economics. He is the ViceChair of the ITS SIG at INFORMS and has chaired many sessions at INFORMS and the Transportation Research Board Conference. His email is <ukkuss@rpi.edu>.

WAI KIN (VICTOR) CHAN is an Assistant Professor of the Department of Decision Sciences and Engineering Systems at Rensselaer Polytechnic Institute. He received a bachelor's degree and a master's degree in electrical engineering from, respectively, Shanghai Jiao Tong University and Tsinghua University, and a M.S. and Ph.D. degrees in industrial engineering and operations research from University of California, Berkeley. His research interests include discrete-event simulation, agent-based simulation, and their applications in energy markets, social networks, service systems, transportation networks, and manufacturing. His e-mail address is $<$ chanw@rpi.edu>. 